\title{
Radio and Polarization Properties in the Disk and Halo of Edge-On Spirals
}

\author{
M. Dumke and M. Krause
}

Max-Planck-Institut für Radioastronomie, Auf dem Hügel 69, D-53121 Bonn, Germany

\begin{abstract}
We have observed a small sample of edge-on galaxies in total and linearly polarized radio continuum emission at $\lambda 6.2 \mathrm{~cm}$, using the Effelsberg $100 \mathrm{~m}$ telescope. The resulting maps were combined with available VLA data in order to obtain high-sensitivity radio images which have a sufficient angular resolution and do not suffer from the so-called missing-spacing problem.

From an analysis of these images (together with $2.8 \mathrm{~cm}$ and $20 \mathrm{~cm}$ data) we get the following results: 1 . The radio emission perpendicular to the plane can be described by a two-component exponential distribution with scale-heights of $\sim 300 \mathrm{pc}$ and $\sim 1.8 \mathrm{kpc}$, independently of the star forming activity and interaction state. 2. In the disk of the non-interacting galaxies of our sample the thermal fraction of the emission seems to be higher than usually deduced from observations of face-on galaxies. 3 . The fractional polarization $p$ increases with increasing galactic height, reaches a maximum at $h \sim 1-3 \mathrm{kpc}$, and decreases again. In general $p$ is lower in actively star forming galaxies.
\end{abstract}

\section{Introduction}

The distribution of relativistic electrons as well as the morphology of uniform and random magnetic fields in galactic halos are strongly affected by dynamical processes in the underlying disk. During the evolution of a galaxy, ongoing star formation leads to subsequent supernova explosions and the creation of superbubbles. These processes may cause the transport of high-energetic particles and small-scale turbulences away from the star forming regions into the disk-halo interface and to high galactic latitudes. As a consequence, the magnetic field properties as well as the spectral distribution of the emission is expected to be different in galaxies of differing star forming activity.

Edge-on galaxies are ideal candidates for observing these effects using radio continuum and polarization measurements at $\mathrm{cm}$-wavelengths, where the emission is mainly due to relativistic electrons gyrating around magnetic field lines. Especially the combination of observations at different wavelengths provide useful information necessary to understand the various relationships between star-formation in the disk and its consequences on the interstellar medium. 


\section{Combination of Interferometer and Single-Dish Observations}

From February to August 1996 we have observed a small sample of edgeon galaxies, consisting of NGC 891, NGC 3628 , and NGC 4565, at $\lambda 6.2 \mathrm{~cm}$ using the Effelsberg $100 \mathrm{~m}$-telescope. The data were reduced in a standard manner using the NOD2 software package available at the MPIfR Bonn. The resulting maps were cleaned from the sidelobes of the telescope pattern, and the instrumental polarization was removed down to a level of $0.3 \%$.

The spatial resolution of large single-dish telescopes is limited. On the other hand, data from interferometers like the VLA suffer from the so called "missing-spacing problem": because of the lack of visibilities at short baselines they are not sensitive to extended emission. In order to get final maps with both the high resolution of the interferometer data and the short-spacing information provided by the single-dish observations, we combined the maps from the Effelsberg telescope with VLA maps at this wavelength from Sukumar \& Allen (1991), Dumke (1994), and Dumke \& Krause (in prep.). This was done in the Fourier plane using the SDE task IMERG (which is available from the NRAO) for the total power as well as the polarization data (Holdaway 1992). Remaining interferences in the combined maps around strong sources, which limited the dynamic range, could successfully be removed using a Fourier filter technique.

The combined maps including the short-spacing information differ significantly from the VLA maps. It is obvious that the usage of interferometer data alone may lead - and have probably led in many investigations in the past to unreliable results concerning the quantitative and qualitative behaviour of radio and polarization properties. Our results also show that even at $\lambda 20 \mathrm{~cm}$ the absence of short spacing information in the interferometer data may be a serious problem. Hence the single-dish data from the Effelsberg telescope are absolutely necessary to investigate the emission of extended radio sources at cm-wavelengths.

\section{Results and Discussion}

Fig. 1 shows the distributions of the radio emission perpendicular to the major axis in the observed galaxies. For all three objects the distribution could be well fitted by a two-component exponential function, convolved with the beam size. Considering also inclination effects, the scale-heights of both components are similar in all galaxies: $h_{\text {thin disk }} \sim 300 \mathrm{pc}$ and $h_{\text {thick disk }} \sim 1.8 \mathrm{kpc}$, independently of the star forming activity in the underlying disk and the interaction state. Since all three galaxies exhibit a large-scale magnetic field predominantly parallel to the disk (Dumke et al. 1995), the field configuration could possibly be the determinant factor for the emission scale-heights. 


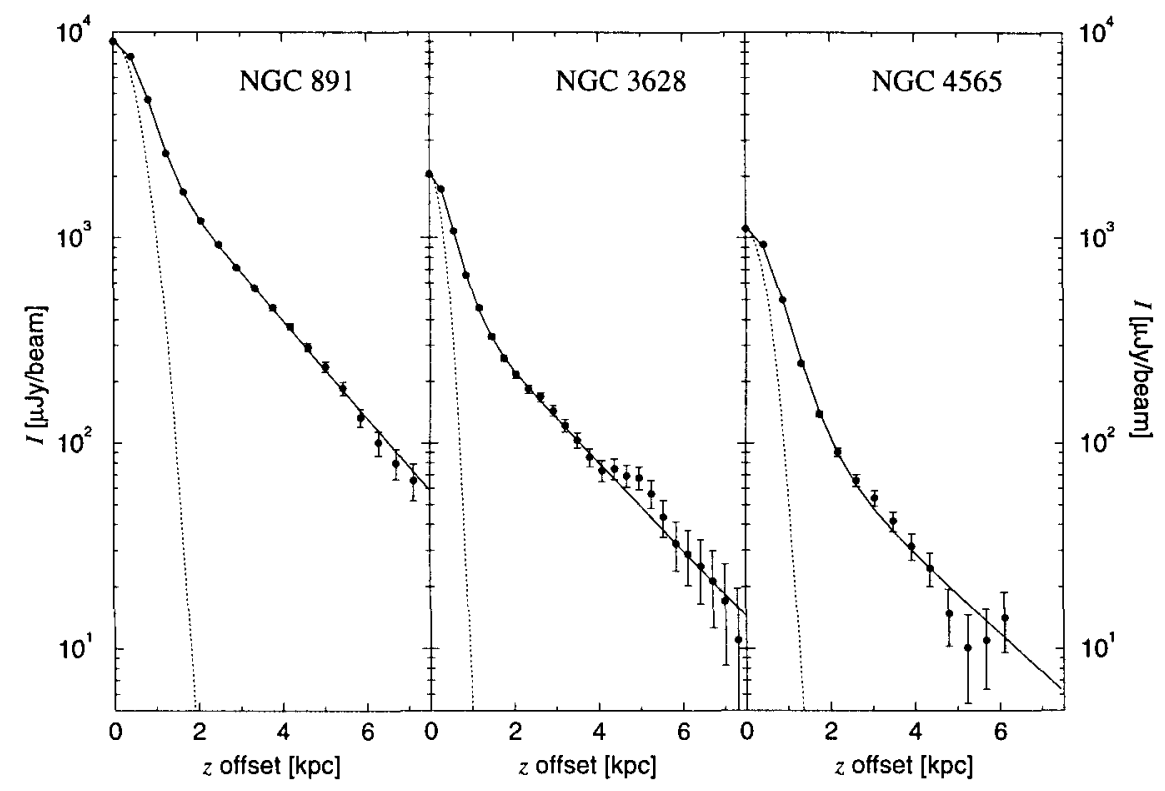

Fig. 1. Distribution of the radio continuum emission perpendicular to the major axis of the galaxies. The dotted lines show the telescope beam, the solid lines the fitted function for a two-component distribution model

Differing results in previous investigations may be caused by a lack of sensitivity/resolution and the lack of large-scale structure information in interferometric data. Strong star forming activity, however, increases the absolute intensities of both components and may be responsible for a relatively high intensity of the thick disk component compared to the thin disk, as it is the case in NGC 891.

The spectral index distribution perpendicular to the plane shows a similar behaviour in all three galaxies: an increase with galactic height $z$. We see (with the available resolution of $2-3 \mathrm{kpc}$ ) no evidence for different transport mechanisms for relativistic electrons. The non-interacting objects NGC 891 and NGC 4565, however, have flatter spectra in the disk and in the halo. Especially the spectra in the disk of these two objects can only be explained with a very high thermal fraction of the total emission, about $50 \%$ at $\lambda 6.2 \mathrm{~cm}$. This is also suggested by a spatial correlation of flat spectra and low fractional polarizations in localized regions in the disk. The spectra observed in faceon galaxies are usually steeper, because those objects must have significant halo emission (which has a steeper spectrum) to yield an observable surface brightness.

The two galaxies NGC 891 and NGC 4565 seem to be very similar, and the first one appears to be just an upscaled version of the latter. On the other 

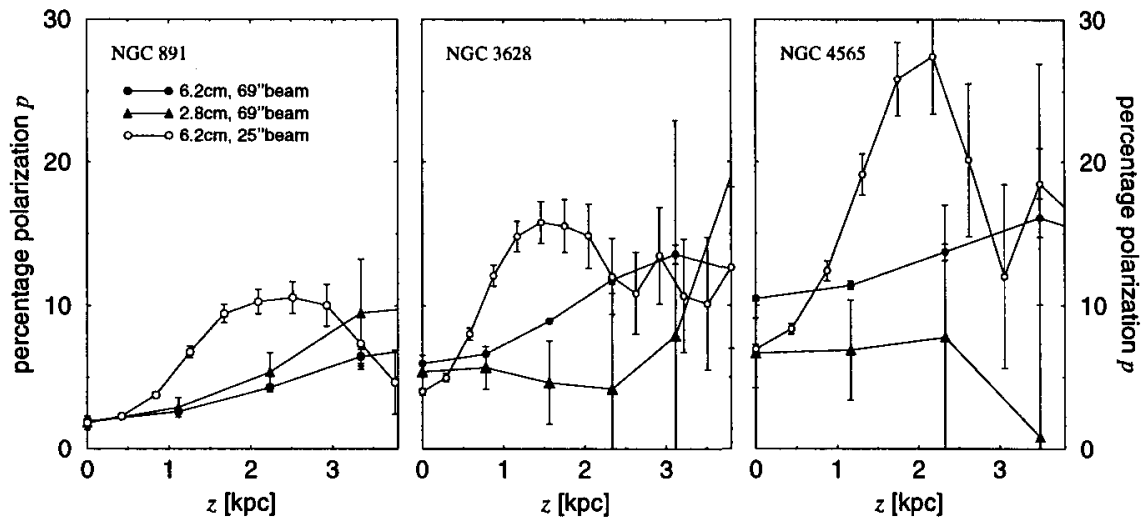

Fig. 2. Degree of polarization in the disk and halo of the observed galaxies for different wavelengths and resolutions

hand the spectral indices in the interacting galaxy NGC 3628 indicate just a very small amount of thermal emission in the galactic disk, and strong star formation seems to be restricted to the central region. Hence the relatively strong thick disk component cannot be due to recent star formation in the thin disk. Possible explanations may be the gravitational interaction with other galaxies and/or that the observed thick disk is a remnant of higher star forming activity in earlier times.

Fig. 2 shows the behaviour of the fractional polarization with increasing distance from the galactic plane, as a function of wavelength and beam size. From the high-resolution $6.2 \mathrm{~cm}$ data (white circles) we find that $p$ increases over the first $\sim 2 \mathrm{kpc}$, but then decreases again. This suggests strong depolarization effects in the disk and in the upper halo of the galaxies. In general, the degree of polarization is highest in NGC 4565, the galaxy with lowest star forming activity, and smallest in NGC 891. This is expected, because star formation leads to enhanced turbulence and more efficient depolarization. At $\lambda 20 \mathrm{~cm}$ (which is not shown in Fig. 2) the polarized intensity is always very weak, due to Faraday depolarization. In case of strong star formation, as in the central region of NGC 3628 and over the whole disk of NGC 891, there is already strong Faraday depolarization at $\lambda 6.2 \mathrm{~cm}$.

\section{References}

Dumke M. (1994): Diploma Thesis, University of Bonn

Dumke M., Krause M., Wielebinski R., Klein U. (1995): A\&A 302, 691

Holdaway M.A. (1992): Mosaicing with Even Higher Dynamic Range, Millimeter

Array Memo No. 73. NRAO, Socorro

Sukumar S., Allen R.J. (1991): ApJ 382, 100 